\title{
A note on almost quasi Yamabe solitons and gradient almost quasi Yamabe solitons
}

\author{
Sujit Ghosh ${ }^{1}$ (D), Uday Chand De ${ }^{2}$ (D), Ahmet Yildiz ${ }^{* 3}$ (D) \\ ${ }^{1}$ Department of Mathematics, Krishnagar Government College, Krishnagar, Nadia, West Bengal, India, \\ Pin-741101 \\ ${ }^{2}$ Department of Pure Mathematics, University of Calcutta, 35, Ballygaunge Circular Road, Kol-700019, \\ West Bengal, India \\ ${ }^{3}$ Education Faculty, Department of Mathematics, Inonu University, Malatya, Turkey
}

\begin{abstract}
In this article, we characterize almost quasi-Yamabe solitons and gradient almost quasiYamabe solitons in context of three dimensional Kenmotsu manifolds. It is proven that if the metric of a three dimensional Kenmotsu manifold admits an almost quasi-Yamabe soliton with soliton vector field $W$ then the manifold is of constant sectional curvature -1 , but the converse is not true has been shown by a concrete example, under the restriction $\phi W \neq 0$. Next we consider gradient almost quasi-Yamabe solitons in a three dimensional Kenmotsu manifold.
\end{abstract}

Mathematics Subject Classification (2020). 53C21, 53D10

Keywords. almost quasi-Yamabe solitons, gradient almost quasi-Yamabe solitons, Kenmotsu manifolds

\section{Introduction}

In a complete Riemannian manifold $(M, g)$ the Riemannian metric $g$ admits Yamabe soliton if it satisfies

$$
\frac{1}{2} £_{W} g=(\rho-\lambda) g
$$

for $\lambda \in \mathbb{R}$ and a differentiable vector field $W$ known as soliton vector field, where $£_{W}$ denotes the Lie derivative along $W$ and $\rho$ being the scalar curvature of $M$. Yamabe solitons is a special solution of Yamabe flow. The notion of Yamabe flow has been introduced by Hamilton [7] towards the study of Yamabe metrics on contact Riemannian manifolds. The Yamabe solitons are known as steady, shrinking and expanding according as $\lambda=0, \lambda>0$ and $\lambda<0$ respectively.

Barbosa and Ribeiro [1] introduced the notion of almost Yamabe soliton by generalizing the Yamabe soliton by setting $\lambda$ to be a smooth function. Seko and Maeta [14] gave a complete classification of almost Yamabe soliton in the context of hypersurfaces in Euclidean spaces.

\footnotetext{
*Corresponding Author.

Email addresses: ghosh.sujit6@gmail.com (S. Ghosh), uc_de@yahoo.com (U. C. De), a.yildiz@inonu.edu.tr (A. Yildiz)

Received: 26.08.2020; Accepted: 20.12.2020
} 
The Yamabe soliton is said to be gradient if the soliton vector field $W$ is the gradient of a $C^{\infty}$ function $\tau$ on $M$. For the case of gradient Yamabe soliton, we have

$$
\nabla^{2} \tau=(\rho-\lambda) g
$$

$\nabla^{2}$ being the Hessian operator. Huang and Li [8] generalized the concept of gradient Yamabe soliton by

$$
\nabla^{2} \tau=\frac{1}{m} d \tau \otimes d \tau+(\rho-\lambda) g
$$

where $m$ is positive constant and $\lambda \in \mathbb{R}$, and named quasi-Yamabe gradient soliton. It is obvious that for $m \rightarrow \infty$ the gradient quasi-Yamabe soliton renders to gradient Yamabe soliton.

In the year 2017, Pirhadi and Razavi [12] studied gradient almost quasi-Yamabe soliton by setting $\lambda$ to be a smooth function. Recently, Chen [4] has considered almost quasiYamabe solitons on almost cosymplectic manifolds. According to Chen, a Riemannian metric $g$ admits almost quasi-Yamabe(AQY) soliton if there exists a smooth function $\lambda$, a $C^{\infty}$ vector field $W$ and a positive constant $m$ such that

$$
\frac{1}{2} £_{W} g=\frac{1}{m} W^{b} \otimes W^{b}+(\rho-\lambda) g
$$

holds, $W^{b}$ being the 1-form associated to $W$. The (AQY) metric is closed if the 1-form $W^{b}$ is closed, whereas trivial if $W=0$. Furthermore, for $m \rightarrow \infty$, the equation (1.2) gives almost Yamabe soliton. Moreover, the preceding equation gives (AQY) gradient soliton $(g, \tau, m, \lambda)$ for $W=D \tau$.

The connected almost contact metric (a.c.m) manifolds with automorphism groups of maximal dimensions has been classified in the following three classes by Tanno [15] in 1969:

$A$. Normal homogeneous contact Riemannian manifolds admitting constant $\phi$-holomorphic sectional curvature for $K(\zeta, U)>0$;

$B$. Global Riemannian product of a line or a circle and a Kählerian manifold with constant holomorphic sectional curvature for $K(\zeta, U)=0$;

$C$. A wraped product space $\mathbb{R} \times_{\lambda} \mathbb{C}^{n}$ if $K(\zeta, U)<0$;

$K(\zeta, U)$ being the sectional curvature of the plane section which contains the Reeb vector field $\zeta$ and the arbitrary smooth vector field $U$.

The manifold of the class $A$ were individualized by some tensor equations and have Sasakian structure. The class $B$ were individualized by some tensor relations which admits a cosymplectic structure. Kenmotsu [10] obtained some tensor equations to characterize the manifolds of the class $C$ in 1972 and this type of manifolds are named as Kenmotsu manifolds. Kenmotsu manifolds has been extensively studied by Pitis in [13].

It is to be noted that in 1872, Lie [11] introduced the concept of contact transformation to study system of differential equations in geometrical point of view. The contact geometry has substantial applications in many areas of physics such as mechanics, optics, control theory, thermodynamics and phase space of dynamical system.

Almost quasi-Yamabe solitons and gradient almost quasi-Yamabe solitons have been considered by Blaga [2] in the context of Riemannian manifolds. Contact metric manifolds admitting Quasi-Yamabe solitons have been considered by Dey and De in [5]. Beside this, Yamabe solitons and quasi-Yamabe solitons have been studied in [6] by Ghosh in Kenmotsu manifolds. Moreover, Wang [16] studied 3-dimensional Kenmotsu manifolds admitting Yamabe solitons and the author proved that if a 3-dimensional Kenmotsu manifold admits 
Yamabe soliton, then the manifold is of constant sectional curvature -1 and the Yamabe soliton is expanding with $\lambda=-6$.

Almost quasi-Yamabe soliton is a natural generalization of Yamabe as well as quasiYamabe soliton. Therefore in this manuscript we set the target to generalize the results which are obtained towards the study of Yamabe and quasi-Yamabe solitons in context of Kenmotsu manifolds in above mentioned works.

The present paper is constructed as follows: Section 2 begins with the discussion of the rudiments of Kenmotsu manifolds. Section 3 is devoted to study three dimensional Kenmotsu manifolds admitting (AQY) solitons and gradient (AQY) solitons. Finally, we construct a non-trivial example of three dimensional Kenmotsu manifold. Precisely we prove the below mentioned theorems:

Theorem 1.1. A three dimensional Kenmotsu manifold admitting a closed (AQY) soliton $(g, W, m, \lambda)$ is a manifold of constant sectional curvature -1 , but the converse is not true, provided $\phi W \neq 0$.

Theorem 1.2. Let a three dimensional Kenmotsu manifold admit gradient (AQY) soliton. Then either the manifold is of constant sectional curvature -1 or the gradient of the (AQY) potential function is point-wise collinear with the characteristic vector field $\zeta$.

\section{Preliminaries}

In this section we discuss the rudiments of Kenmotsu manifolds. An odd dimensional $C^{\infty}$ manifold $M$ is named to be an a.c.m. manifold if it admits a Reeb vector field $\zeta$, a $(1,1)$-tensor field $\phi$, a global 1-form $\eta$ and a Riemannian metric $g$ satisfying [3]:

$$
\begin{aligned}
& \phi^{2}=-I+\eta \times \zeta, \quad \eta(\zeta)=1, \quad \phi \zeta=0, \quad \eta(\zeta)=1, \\
& \eta \circ \phi=0, \quad g(U, \zeta)=\eta(U), \quad g(\phi U, \phi V)=g(U, V)-\eta(U) \eta(U),
\end{aligned}
$$

where $U, V$ are smooth vector fields on $M$. It is to be noted that an a.c.m. structure on a $(2 n+1)$-dimensional Riemannian manifold $M$ may be considered as a reduction of the structure group $M$ to $U(n) \times I$.

The fundamental two form $\Phi$ of a $(2 n+1)$-dimensional a.c.m. manifold $M$ is defined by $\Phi(U, V)=g(U, \phi V)$, where $U, V \in \chi(M)$. An a.c.m. manifold is said to be an almost Kenmotsu manifold if $d \eta=0$ and $d \Phi=2 \eta \wedge \Phi$ (see [9]). An almost Kenmotsu manifold is said to be normal if the tensor $N_{\phi}=[\phi, \phi]+2 d \eta \otimes \zeta$ vanishes, $[\phi, \phi]$ being the Nijenhuis tensor of $\phi$. An almost Kenmotsu manifold is said to be a Kenmotsu manifold if it is normal [9], and condition of normality can be expressed as

$$
\left(\nabla_{U} \phi\right) V=g(\phi U, V) \zeta-\eta(V) \phi U,
$$

$U, V$ being smooth vector fields on $M$. In a $(2 n+1)$-dimensional Kenmotsu manifolds the following relations are satisfied [10]:

$$
\begin{gathered}
\nabla_{U} \zeta=U-\eta(U) \zeta, \\
K(U, V) \zeta=\eta(U) V-\eta(V) U, \\
\left(\nabla_{U} \eta\right) V=g(U, V)-\eta(U) \eta(V), \\
S(U, \zeta)=g(Q \zeta, U)=-2 n \zeta,
\end{gathered}
$$

for $U, V \in \chi(M)$, where $K, S$ and $Q$ denotes the curvature tensor, Ricci tensor and Ricci operator respectively.

Again, in a three dimensional Riemannian manifold, we have

$$
\begin{aligned}
K(U, V) Z= & g(V, Z) Q U-g(U, Z) Q V+S(V, Z) U \\
& -S(U, Z) V-\frac{\rho}{2}\{g((V, Z) U-g(U, Z) V)\}
\end{aligned}
$$


for any vector fields $U, V, Z$ in $M^{3}$. (2.7) yields

$$
S(U, V)=\left(\frac{\rho}{2}+1\right) g(U, V)-\left(\frac{\rho}{2}+3\right) \eta(U) \eta(V) .
$$

Making use of (2.8) in (2.7), we have

$$
\begin{aligned}
K(U, V) Z= & \left(\frac{\rho}{2}+2\right)\{g(V, Z) U-g(U, Z) V\} \\
& -\left(\frac{\rho}{2}+3\right)\{g(V, Z) \eta(U) \zeta-g(U, Z) \eta(V) \zeta \\
& +\eta(V) \eta(Z) U-\eta(U) \eta(Z) V\} .
\end{aligned}
$$

To produce our main theorems, we need the following Lemmas:

Lemma 2.1. In 3-dimensional Kenmotsu manifold $(M, \phi, \zeta, \eta, g)$ the following relation holds:

$$
\nabla_{\zeta} D \rho=4(\rho+6) \zeta
$$

Proof. In [16], Wang proved that in a 3-dimensional Kenmotsu manifold $\zeta(\rho)=-2(\rho+6)$. By virtue of $g\left(\nabla_{U} D \rho, V\right)=g\left(\nabla_{V} D \rho, U\right)$ from $\zeta(\rho)=-2(\rho+6)$ we obtain (2.10). This completes the proof.

Lemma 2.2 ([4]). For any vector fields $U, V$ on $M$, for a gradient (AQY) soliton $(M, g, \tau, m, \lambda)$, we have

$$
\begin{aligned}
K(U, V) D \tau= & \frac{\rho-\lambda}{m}[(V \tau) U-(U \tau) V] \\
& +(U(\rho-\lambda)) V-(V(\rho-\lambda)) U,
\end{aligned}
$$

$D$ being the gradient operator of $g$.

\section{Proof of the main theorems}

Proof of the Theorem 1.1. Let $M$ be a three dimensional Kenmotsu manifold admitting a closed (AQY) soliton $(g, W, m, \lambda)$. Since $W^{b}$ is closed, equation (1.2) is equivalent to

$$
\nabla_{V} W=(\rho-\lambda) V+\frac{1}{m} g(W, V) W
$$

Performing covariant derivative of (3.1) along $U$, we obtain

$$
\begin{aligned}
\nabla_{U} \nabla_{V} W= & (U(\rho-\lambda)) V+(\rho-\lambda) \nabla_{U} V \\
& +\frac{1}{m}\left\{g\left(\nabla_{U} W, V\right)+g\left(W, \nabla_{U} V\right)\right\} W \\
& +\frac{1}{m} g(W, V) \nabla_{U} W .
\end{aligned}
$$

Interchanging $U$ and $V$ in the preceding equation yields

$$
\begin{aligned}
\nabla_{V} \nabla_{U} W= & (V(\rho-\lambda)) U+(\rho-\lambda) \nabla_{V} U \\
& +\frac{1}{m}\left\{g\left(\nabla_{V} W, U\right)+g\left(W, \nabla_{V} U\right)\right\} W \\
& +\frac{1}{m} g(W, U) \nabla_{V} W .
\end{aligned}
$$

Again, putting $V$ instead of $[U, V]$ in (3.1) yields

$$
\nabla_{[U, V]} W=(\rho-\lambda)[U, V]+\frac{1}{m} g(W,[U, V]) W .
$$


Using (3.2)-(3.4) in the well known formula $K(U, V) W=\nabla_{U} \nabla_{V} W-\nabla_{V} \nabla_{U} W-\nabla_{[U, V]} W$, we reveal that

$$
\begin{aligned}
K(U, V) W= & (U(\rho-\lambda)) V-(V(\rho-\lambda)) U \\
& +\frac{\rho-\lambda}{m}\{g(W, V) U-g(W, U) V\} .
\end{aligned}
$$

Taking inner product of $(3.5)$ with $\zeta$, we get

$$
\begin{aligned}
g(K(U, V) W, \zeta)= & \left\{(U(\rho-\lambda))-\frac{\rho-\lambda}{m} g(U, W)\right\} \eta(V) \\
& -\left\{(V(\rho-\lambda))-\frac{\rho-\lambda}{m} g(V, W)\right\} \eta(U) .
\end{aligned}
$$

On the other hand, in view of (2.4) we infer that

$$
g(K(U, V) W, \zeta)=g(U, W) \eta(V)-g(V, W) \eta(U) .
$$

Comparing the equations (3.6) and (3.7) yields

$$
\begin{aligned}
& \left\{(U(\rho-\lambda))-\frac{\rho-\lambda}{m} g(U, W)\right\} \eta(V) \\
& -\left\{(V(\rho-\lambda))-\frac{\rho-\lambda}{m} g(V, W)\right\} \eta(U) \\
= & g(U, W) \eta(V)-g(V, W) \eta(U) .
\end{aligned}
$$

Replacing $U$ and $V$ by $\phi U$ and $\zeta$ respectively and using (2.1) entails that

$$
\phi D(\rho-\lambda)=\left(1+\frac{\rho-\lambda}{m}\right) \phi W .
$$

On the other hand, contracting (3.5) over $V$ and utilizing (2.8), we infer that

$$
\left[\frac{\rho}{2}+1-\frac{2(\rho-\lambda)}{m}\right] W-\left(\frac{\rho}{2}+3\right) \eta(W) \zeta=-2 D(\rho-\lambda) .
$$

Applying $\phi$ on the both sides of (3.10) and using (2.1) yields

$$
\left[\frac{\rho}{2}+1-\frac{2(\rho-\lambda)}{m}\right] \phi W=-2 \phi D(\rho-\lambda) .
$$

In view of (3.9) and (3.11), we get

$$
\left(\frac{\rho}{2}+3\right) \phi W=0
$$

If we consider $\phi W \neq 0$, the preceding equation implies that $\rho=-6$. For $\rho=-6$ the equation (2.9) delivers $K(U, V) Z=-\{g(V, Z) U-g(U, Z) V\}$. Therefore we have the manifold is of constant sectional curvature -1 .

In order to show that the converse of the above result is not true here we mention the following example:

We consider a three dimensional manifold $M=\left\{(x, y, z) \in \mathbb{R}^{3}\right\},(x, y, z)$ being the standard coordinates of $\mathbb{R}^{3}$. Let us consider the vector fields $e_{1}, e_{2}, e_{3}$ on $M$ be such that

$$
\left[e_{1}, e_{2}\right]=0, \quad\left[e_{2}, e_{3}\right]=e_{2}, \quad\left[e_{1}, e_{3}\right]=e_{1} .
$$

We define the Riemannian metric $g$ by $g\left(e_{i}, e_{j}\right)=\delta_{i j}$, for $i, j=1,2,3$, the 1-form $\eta$ by $\eta(U)=g\left(U, e_{3}\right)$ and the $(1,1)$ tensor field $\phi$ by $\phi\left(e_{3}\right)=0, \phi\left(e_{2}\right)=e_{1}, \phi\left(e_{1}\right)=-e_{2}$, where $U \in \chi(M)$.

Then using the well known Koszul's formulae given by $2 g\left(\nabla_{U} V, Z\right)=U g(V, Z)+$ $V g(Z, U)-Z g(U, V)-g(U,[V, Z])-g(V,[U, Z])+g(Z,[U, V])$, we calculate the following:

$$
\begin{aligned}
& \nabla_{e_{1}} e_{3}=e_{1}, \nabla_{e_{1}} e_{2}=0, \nabla_{e_{1}} e_{1}=-e_{3}, \\
& \nabla_{e_{2}} e_{3}=e_{2}, \nabla_{e_{2}} e_{2}=-e_{3}, \nabla_{e_{2}} e_{1}=0, \\
& \nabla_{e_{3}} e_{3}=0, \nabla_{e_{3}} e_{2}=0, \nabla_{e_{3}} e_{1}=0 .
\end{aligned}
$$


In view of above computations it is clear that the manifold satisfies $\nabla_{U} \zeta=U-\eta(U) \zeta$ for $\zeta=e_{3}$. Hence the manifold $M$ is a three dimensional Kenmotsu manifold.

Again, using the formula $K(U, V) Z=\nabla_{U} \nabla_{V} Z-\nabla_{V} \nabla_{U} Z-\nabla_{[U, V]} Z$, we calculate the following independent non-vanishing components of the curvature tensor $K$ :

$$
\begin{array}{ll}
K\left(e_{1}, e_{2}\right) e_{2}=-e_{1}, & K\left(e_{1}, e_{3}\right) e_{3}=-e_{1} \\
K\left(e_{2}, e_{1}\right) e_{1}=-e_{2}, & K\left(e_{2}, e_{3}\right) e_{3}=-e_{2} \\
K\left(e_{3}, e_{1}\right) e_{1}=-e_{3}, & K\left(e_{3}, e_{2}\right) e_{2}=-e_{3} .
\end{array}
$$

From the above expressions of curvature tensors we derive the non-vanishing components of the Ricci tensor as given below:

$$
S\left(e_{1}, e_{1}\right)=-2, \quad S\left(e_{2}, e_{2}\right)=-2, \quad S\left(e_{3}, e_{3}\right)=-2
$$

and therefore the scalar curvature of this manifold $\rho=\sum_{i=1}^{3} S\left(e_{i}, e_{i}\right)=-6$.

Let $U=u_{1} e_{1}+u_{2} e_{2}+u_{3} e_{3}, V=v_{1} e_{1}+v_{2} e_{2}+v_{3} e_{3}$ and $Z=z_{1} e_{1}+z_{2} e_{2}+z_{3} e_{3}$. Then it is easy to verify that $K(U, V) Z=-[g(V, Z) U-g(U, Z) V]$ i.e., the manifold is of constant sectional curvature -1 .

Now if we consider an arbitrary soliton vector field $W=w_{1} e_{1}+w_{2} e_{2}+w_{3} e_{3}$, then we see that $\nabla_{e_{1}} W=-w_{1} e_{3}+w_{3} e_{1}$ and $(\rho-\lambda) e_{1}+\frac{1}{m} g\left(W, e_{1}\right) W=-(6+\lambda) e_{1}-\frac{1}{m} w_{1}\left\{w_{1} e_{1}+\right.$ $\left.w_{2} e_{2}+w_{3} e_{3}\right)$. Therefore $\nabla_{e_{1}} W \neq(\rho-\lambda) e_{1}+\frac{1}{m} g\left(W, e_{1}\right) W$ for any choice of $\lambda, m$ and $W$. Hence the manifold can not admit an almost quasi-Yamabe soliton although it is a 3 dimensional Kenmotsu manifold with constant sectional curvature -1 . This finishes the proof.

Now, for $m \rightarrow \infty$ and $\lambda=$ constant the (AQY) soliton becomes a Yamabe soliton. Then from (3.9) and (3.11), we obtain

$$
\phi D \rho=\phi W
$$

and

$$
\left[\frac{\rho}{2}+1\right] \phi W=-2 \phi D \rho .
$$

respectively. (3.13) and (3.14) yields

$$
\left(\frac{\rho}{2}+3\right) \phi W=0
$$

Hence either $\rho=-6$ or $\phi W=0$. Using $\rho=-6$ in (2.9) yields $K(U, V) Z=-[g(V, Z) U-$ $g(U, Z) V]$ i.e., the manifold is of constant sectional curvature -1 . On the other hand, for $\phi W=0$, we have from (3.13) that $\phi D \rho=0$. Applying $\phi$ on preceding relation and using $(2.1)$ and $\zeta \rho=-2(\rho+6) \zeta$, we have

$$
D r=-2(\rho+6) \zeta .
$$

Taking covariant differentiation of (3.16) along $U$ and making use of (3.16) yields

$$
\nabla_{U} D \rho=6(\rho+6) \eta(U) \zeta-2(\rho+6) U .
$$

Again, for Yamabe soliton we have the soliton vector field $W$ is conformal with conformal co-efficient $\varrho=\rho-\lambda$. For conformal vector field, we recall the following formula [17]

$$
\begin{aligned}
\left(£_{W} K\right)(U, V) Z= & g\left(\nabla_{U} D \varrho, Z\right) V-g\left(\nabla_{V} D \varrho, Z\right) U \\
& +g(U, Z) \nabla_{V} D \varrho-g(V, Z) \nabla_{U} D \varrho .
\end{aligned}
$$

For $m \rightarrow \infty$ and $\lambda=$ constant, we have from (1.2),

$$
\left(£_{W} \eta\right) U-g\left(U, £_{W} \zeta\right)=2(\rho-\lambda) \eta(U) .
$$


Taking $U=\zeta$ in the foregoing equation yields $g\left(£_{W} \zeta, \zeta\right)=-(\rho-\lambda)$. Taking Lie derivative of (2.4) along $W$ we use (3.18) and (3.19) to achieve

$$
\begin{aligned}
& \left(£_{W} K\right)(U, V) £_{W} \zeta+g\left(\nabla_{U} D \rho, \zeta\right) V-g\left(\nabla_{V} D \rho, \zeta\right) U+\eta(U) \nabla_{V} D \rho \\
& -\eta(V) \nabla_{U} D \rho=g\left(U, £_{W} \zeta\right) V-g\left(V, £_{W} \zeta\right) U+2(\rho-\lambda)[\eta(U) V-\eta(V) U] .
\end{aligned}
$$

Setting $V=\zeta$ in (3.20) and making use of (2.4), (2.10) and (3.20), we deduce

$$
\nabla_{U} D \rho=2(3 \rho+\lambda+24) \eta(U) \zeta-2(\rho+\lambda+12) U .
$$

Comparing (3.17) and (3.21), we at once obtain $\lambda=-6$ i.e., the soliton is expanding. Therefore we state the following:

Corollary 3.1. Let a three dimensional Kenmotsu manifold admit Yamabe soliton. Then the manifold is either of constant sectional curvature -1 or the soliton is expanding with $\lambda=-6$.

The above corollary has been proved in [16] by Wang.

Proof of the Theorem 1.2. Let us assume that the metric of a three dimensional Kenmotsu manifold admits gradient (AQY) soliton. Taking inner product of (2.11) with $\zeta$, we obtain

$$
\begin{aligned}
g(K(U, V) D \tau, \zeta)= & \frac{\rho-\lambda}{m}\{(V \tau) \eta(U)-(U \tau) \eta(V)\} \\
& +(U(\rho-\lambda)) \eta(V)-(V(\rho-\lambda)) \eta(U) .
\end{aligned}
$$

In view of (2.4) we have

$$
g(K(U, V) D \tau, \zeta)=[(U \tau) \eta(V)-(V \tau) \eta(U)] .
$$

In view of (3.22) and (3.23), we have

$$
\begin{aligned}
& \frac{\rho-\lambda}{m}\{(V \tau) \eta(U)-(U \tau) \eta(V)\} \\
& +(U(\rho-\lambda)) \eta(V)-(V(\rho-\lambda)) \eta(U) \\
= & {[(U \tau) \eta(V)-(V \tau) \eta(U)] . }
\end{aligned}
$$

Replacing $U, V$ by $\phi U$ and $\zeta$ respectively in the foregoing equation and making use of (2.1), we reveal that

$$
\left(1+\frac{\rho-\lambda}{m}\right)(\phi U) \tau=(\phi U)(\rho-\lambda) .
$$

On the other hand, contracting (2.11) with respect to $V$ and making use of (2.8), we infer that

$$
2(\phi U)(\rho-\lambda)=\frac{2(\rho-\lambda}{m}(\phi U) \tau-\left(\frac{\rho}{2}+1\right)(\phi U) \tau .
$$

Comparing (3.25) and (3.26), we have

$$
\left(\frac{\rho}{2}+3\right)(\phi U) \tau=0 .
$$

Therefore, either $\rho=-6$ or $(\phi U) \tau=0$.

Case (i): For $\rho=-6$, the equation (2.9) gives $K(U, V) Z=-\{g(V, Z) U-g(U, Z) V\}$ and the from (2.8) we have $S(U, V)=-2 g(U, V)$. Therefore in this case the manifold is Einstein and the sectional curvature of the manifold is -1 .

Case (ii): For $(\phi U) \tau=0$, we apply $\phi$ and use $(2.1)$ to get $(U \tau)=(\zeta \tau) \zeta$. Therefore we conclude that the gradient of the (AQY) potential function $\tau$ is pointwise collinear with the characteristic vector field $\zeta$. Hence the proof is completed. 


\section{References}

[1] E. Barbosa and E. Ribeiro, On conformal solutions of the Yamabe flow, Arch. Math. 101, 79-89, 2013.

[2] A.M. Blaga, A note on warped product almost quasi-Yamabe solitons, Filomat 33, 2009-2016, 2019.

[3] D.E. Blair, Contact manifolds in Riemannian geometry, Lect. Notes Math. 509, 1976.

[4] X. Chen, Almost quasi-Yamabe solitons on Almost cosymplectic manifolds, Int. J. Geom. Methods Mod. Phys. 17, 2050070, 2020.

[5] C. Dey and U.C. De, A note on quasi-Yamabe solitons on contact metric manifolds, J. Geom. 111, 1-7, 2020.

[6] A. Ghosh, Yamabe soliton and quasi Yamabe soliton on Kenmotsu manifold, Math. Slovaca 70, 151-160, 2020.

[7] R.S. Hamilton, The Ricci flow on surfaces, Mathematics and General Relativity, Contemp. Math. 71, 237-262, 1988.

[8] G. Huang and H. Li, On a classification of the quasi-Yamabe gradient solitons, Methods. Appl. Anal. 21, 379-390, 2014.

[9] D. Janssens and L. Vanhecke, Almost contact structures and curvature tensors, Kodai Math. J. 4, 1-27, 1981.

[10] K. Kenmotsu, A class of almost comtact Riemannian manifolds, Math. Ann. 219, 93-103, 1972.

[11] S. Lie, Theorie der Transformationgruppen, 2, Leipzig, Tenbuer, 1890.

[12] V. Pirhadi and A. Razavi, On the almost quasi-Yamabe solitons, Int. J. Geom. Methods Mod. Phys. 14, 1750161, 2017.

[13] G. Pitis, A remark on Kenmotsu manifolds, Bull. Univ. Brasov Ser. C. 30, 31-32, 1988.

[14] T. Seko and S. Maeta, Classifications of almost Yamabe solitons in Euclidean spaces, J. Geome. Phys. 136, 97-103, 2019.

[15] S. Tanno, The automorphism groups of almost contact Riemannian manifolds, Tohoku Math. J. 21, 21-38, 1969.

[16] Y. Wang, Yamabe solitons on three-dimensional Kenmotsu manifolds, Bull. Belg. Math. Soc. Simon Stevin 23, 345-355, 2016.

[17] K. Yano, Integral formulas in Riemannian geometry, Marcel Dekker, New York, 1970. 